\title{
Neurological Markers of Maladaptive Brain Activity in Fibromyalgia and their Relationship with Treatment Effectiveness
}

\author{
Elia Valentini ${ }^{1}$
}

Chronic pain (CP) is estimated to affect at least one-third of the population in the United Kingdom. Fibromyalgia (FM) is one of the most disabling CP conditions. Epidemiological research suggests its global prevalence to be between 2-8\%. The unknown pathogenesis, lack of biological markers to monitor its development, and lack of successful treatment make FM a crucial target of pre-clinical research.

The goal of this project is twofold. The project aims to 1) identify robust neurological markers (i.e., electrochemical brain activity) by applying a combination of advanced electroencephalography (EEG) signal processing (i.e., functional connectivity of oscillatory activity) and neuroinflammatory (NI) responses (i.e., estimation of pro-inflammatory cytokines intake), through which 2) characterizing successfully and unsuccessfully treated FM patients (compared to age-matched healthy controls). These measures, seldom combined, have been successfully applied to the study of psychiatric conditions and sleep. Crucially, the identification of neurological markers at rest and during arousing sensory stimulation will allow us to estimate the relationship between these neurological markers and treatment effectiveness. This proposal is important because it aims to generate a robust pre-clinical neurological tool to identify FM and its relationship with measures of treatment effectiveness. The successful identification of neurological markers will improve the assessment of the development of maladaptive changes in FM and will kick-start further research on treatment effectiveness.

This project is of great medical relevance as it will identify pathological signatures of FM that can then inform research on etiology and treatment of this condition.

Keywords fibromyalgia, pain, EEG, cytokines, neuroinflammation, brain

${ }^{1}$ Department of Psychology and Centre for Brain Science, University of Essex

Received 10 November, 2021 Accepted 22 November, 2021 Published 31 December, 2021 Issued 3 March, 2022

Correspondence evalent@essex.ac.uk

Grant Application to: Academy of Medical Sciences Springboard Round 42018 SBF004\1012

License (c) (i)

This article is licensed under the Creative Commons Attirbution 4.0 (CC-BY 4.0) license, which allows you to copy, redistribute, transform and build upon the article and all its contents in any medium ar format for any medium or format for any purpose, provided that appropriate credit is given

(c) Valentini 2021

Check for updates

\section{Research proposal}

The institution of employment at time of application

University of Essex

Prospective host institution

University of Essex

Main field

Neuroscience

Other fields

Pain

Description of the proposed research

Overall aim and key objectives

Pain is a major burden for our society. In the United Kingdom, chronic pain is estimated to affect between one-third and one-half of the population. This impressive figure is likely to increase with the progressive ageing of the population [1]. A recent survey revealed that $19 \%$ of the European population have experienced moderate or severe pain for at least six months duration and at least twice a week. About $40 \%$ of these people report insufficient pain treatment, both pharmacological and non-pharmacological [2].

Fibromyalgia (FM) is a chronic pain syndrome affecting the central, autonomic, endocrine, and immune systems, often leading to an intense state of suffering and disability. Epidemiological research suggests its global prevalence to be between 2-8\% with a predominant female/male ratio [3]. FM diagnosis is currently accomplished through clinical assessment, as no validated biological markers have been identified. Its diagnosis relies on the assessment of subjective dimensions such as pain, fatigue, and sleep quality using the Symptom Severity (SS) and Widespread Pain Index (WPI) [4]. As a result, there is large variability between diagnoses [5]. Research suggests that central nervous system sensitization is considered the hallmark of the pathological trajectory of FM, leading to an increased gain in pain and sensory processing [6].

Neuroimaging studies using functional magnetic resonance imaging show altered brain communication between cerebral regions in FM patients, 
namely intrinsic resting state connectivity $[7,8]$. Further evidence points to inflammatory mechanisms triggered by the activation of mast cells [9]. Current targets of investigation in FM are IL-1, IL-6, and IL-8 and the tumor necrosis factor (TNF) [10]. Despite increased investigation into the relationship between increase in cytokines synthesis and increased excitability of the brain [11], no research project has studied them in combination with EEG activity in FM.

Importantly, many FM patients report dissatisfaction with the available treatments [12]. Management strategies for FM vary and the effect size of most treatments is modest [13]. Therefore, not only is there a lack of diagnostic tools to effectively identify FM, but there is also high variability and limited effectiveness of its treatment. By "treatment effectiveness", the project refers to how beneficial a FM treatment is in everyday life compared to no treatment, assessed by a series of outcome measures, such as pain, fatigue, mood and anxiety, sleep quality, daily functioning, and well-being [14]. An effective treatment is one that is likely to reduce/improve the aforementioned symptoms. It follows that there may be substantial differences in brain and neuroinflammatory (NI) correlates between healthy and FM individuals, as well as between FM individuals who are experiencing an effective treatment compared to those who have not had a successful therapy. Neither issue has received enough investigation so far.

\section{Research plan}

Design The project addresses the following question: Can we exploit functional connectivity of oscillatory EEG activity and pro-inflammatory cytokines intake as pre-clinical neurological markers and treatment effectiveness in FM? In other words, this project aims to extend existing knowledge on abnormal brain processing and enhanced NI activity in FM. Another element of this project's originality comes from our attempt to provide a characterization of neurological patterns in FM individuals undergoing effective vs. ineffective treatment (compared to age-matched healthy controls). Thus, there will be two specific objectives: 1) to identify distinct EEG and NI markers of FM; 2) to characterize the EEG and NI profile associated with treatment effectiveness in FM. FM patients will be assigned to two different outcome groups based on scores in pain, fatigue, mood and anxiety, sleep quality, daily functioning, and well-being. The project will then measure their neurological trajectories prior to and 3 months after the start of their treatment (see tasks in Fig. 1 timeline) using a combination of advanced EEG signal processing and $\mathrm{NI}$ responses. These measures, seldom combined, have been successfully applied to the study of psychiatric conditions and sleep. In the meantime, EEG responses will also be recorded in a group of healthy age-matched individuals. Therefore, the research design will consist of three groups: FM patients with effective treatment (Feff), FM patients with ineffective treatment (Fineff), and age-matched healthy controls (Ctrl). Treatment effectiveness will be assessed based on reductions in current symptoms compared to the pre-treatment. By doing so, the project essentially asks whether discriminatory patterns of EEG and $\mathrm{NI}$ can be detected not only between Ctrl and FM patients, but also between Feff and Fineff.

It is estimated that at least 20 participants per each experimental group will enter the study (in line with a power estimate based on previous neuroimaging studies). Participants will be all female (because of the large predominance of the disease in the female population) and selected on the basis of stringent exclusion criteria [7]. The control group of healthy age-matched individuals $(n=30)$ will outnumber the experimental groups to increase the accuracy of the findings.

\section{Methodology}

Self report measures The main criterion for participation will be a diagnosis of FM and the final score in the SS and WPI. Participants who do not meet the inclusion criterion of WPI $>7$ and SS $>5$ or WPI between 3-6 and SS > 9 will be not allowed to enter the study, as they would not clinically classify as FM patients. Patients who enter the study will then be submitted to a series of measures. The project will use the Fibromyalgia Impact Questionnaire (FIQ), which is a multidimensional self-administered instrument that covers the following dimensions: physical functioning (11 items), well-being (1 item), work situation (2 items), pain (1 item), fatigue/sleep (2 items), stiffness ( 2 items), and psychological symptoms (2 items). The Patient Health Questionnaire (PHQ-9) will be used to investigate current disposition to depressive symptoms [15] and the General Anxiety Disorder questionnaire [16] will be used to assess disposition to anxiety. In addition, participants will complete the Pain Catastrophizing Scale (PCS) [17], a questionnaire that measures the cognitive/emotional attitude towards pain, and the WarwickEdinburgh Mental Well-being Scale (WEMWBS) [18] to quantify well-being.

EEG recording and data analysis I have extensive experience in EEG techniques and pain research $[19,20]$. The project will take advantage of my collaboration with Fibromyalgia Research UK to recruit FM patients and with the molecular biology lab led by Dr. Metodiev to extract cytokines from participants' blood samples and quantify them for each participant. As FM is known to be associated with abnormal processing in both rest and stimulation states [6], EEG activity will be measured at rest and 


\begin{tabular}{|c|c|c|c|c|c|c|c|c|c|c|c|c|c|c|c|c|c|c|c|c|c|c|c|c|}
\hline & \multicolumn{24}{|c|}{ Project Timeline } \\
\hline Years & \multicolumn{12}{|c|}{1} & \multicolumn{12}{|c|}{2} \\
\hline Months & 1 & 2 & & 4 & 5 & 6 & $7 \mid$ & 8 & 9 & 10 & 11 & 12 & 13 & 14 & 15 & 16 & 17 & 18 & $19 \mid$ & $20 \mid$ & 21 & 22 & 23 & 24 \\
\hline \multicolumn{25}{|l|}{ Tasks } \\
\hline \multicolumn{25}{|l|}{$\mathrm{T} 1$} \\
\hline \multicolumn{25}{|l|}{ T2.1 } \\
\hline \multicolumn{25}{|l|}{$\mathrm{T} 2.2$} \\
\hline \multicolumn{25}{|l|}{$\mathrm{T} 3$} \\
\hline \multicolumn{25}{|l|}{ T4 } \\
\hline Project Milestones & & & & प1 & & & & & & प2 & & M3 & & & & & & & & & & & & M4 \\
\hline
\end{tabular}

Figure 1 Project timeline. The scientific objectives of the project will be achieved through 4 Tasks (T) and verified at 4 Milestones (M). The first month of the project will involve the PI and the PDRA in collecting relevant clinical data and self-report questionnaire scores of Fibromyalgia (FM) patients who just started a therapy (T1, red). These will be collected again after 3 months to determine which of the patients are benefitting from their therapy (i.e., showing reduction in symptoms). Based on this assessment, participants will be assigned to either the effective treatment (Feff) group, or to the ineffective treatment (Fineff) group (M1). Both groups will undergo a first experimental session (T2.1, green) whereby symptoms will be recorded, blood sampling for extraction of neuroinflammatory (NI) indexes will be performed, and electroencephalography (EEG) data will be obtained at month 10 (M2). Symptoms and EEG measurements will also be collected in age-matched healthy controls (Ctrl) which will start in parallel with T2.1 (T3, orange) but will proceed at lower priority and end at month 12. A minimum lapse of 3 months will be interleaved before a FM patient goes through a second session of symptoms, NI, and EEG data collection (T2.2, light green), wherein the same staff will be involved. T2.2 will initially be paced slowly because of the overlapping T2.1 and T3. The last task (T4, blue) will start at month 16 and will consist of statistical analysis of self-report, NI, and EEG data for the three groups, and preparation of the findings for dissemination in the shape of conference presentations and scientific articles (M4). T4 will involve the PDRA, the PI and the scientific collaborator (Dr. Metodiev).

during repetition of vibrotactile stimuli at both TO and T1. This approach, while providing us with a measure of central sensitization of the somatosensory system, will not cause pain. Intrinsic oscillatory activity with eyes opened and eyes closed at rest will be analyzed, as well as stimulus-related activity. Both traditional power spectra analysis and functional connectivity approaches will be used.

More precisely, I will test whether 1) patients who did not achieve an effective treatment will show reduced EEG synchronization and increased frontoparietal connectivity compared to healthy controls and patients who obtained an effective treatment; 2) patients who did achieve an effective treatment will show increased EEG synchronization and decreased mid-cingulate/premotor connectivity (see Fig. 2 for preliminary data) on T1 compared to T0 (and vice versa for patients who did not obtain effective treatment); 3) this effect will be predicted by increased cytokine concentration for patients who did not achieve effective treatment (and predicted by reduced cytokine intakes in patients who obtained an effective treatment).

Cytokines extraction and analysis Cytokines IL-1, IL6, and IL-8 and TNF will be obtained through blood sampling before each EEG session. Symptoms will be collected before, during, and after the experimental session. In particular, the project will analyze the relationship between self-report measures (e.g., pain), EEG magnitude, and connectivity in different frequencies and cytokines.

Commercial enzyme-linked immuno-sorbent assay (ELISA) kits will be used to quantify the cytokines according to manufacturers' instructions. An example is the IL-1 kit from Thermo Fisher. ELISA is a sensitive and robust method for quantitative analysis of low-level biomarkers in blood and plasma samples. More specifically, the project will use the sandwich ELISA method, which uses two antigen-specific antibodies-one to capture the target molecule and the other to generate the signal for quantification. Commercial sandwich ELISA kits are available for all of the proposed cytokines. They are well-validated and will provide the necessary level of sensitivity and specificity for the study. The cytokine analyses will be performed in Dr Metodiev's lab, which is equipped with all necessary instruments and staffed by personnel experienced in biochemical analyses and proteomics.

\section{Knowledge utilization}

The successful identification of neurological markers will pave the way for experimental studies aimed at disentangling the effects of both pharmacological and non-pharmacological treatments on these neurological measures. The novel combination of EEG and NI measures in this study will improve the assessment of development of maladaptive changes in FM and inform research on treatment effectiveness. The output of this research will be published in reputable journals such as e.g., Cortex, eLife, Frontiers in Neuroscience, Journal of Neuroinflammation, Neurolmage, and Pain. 

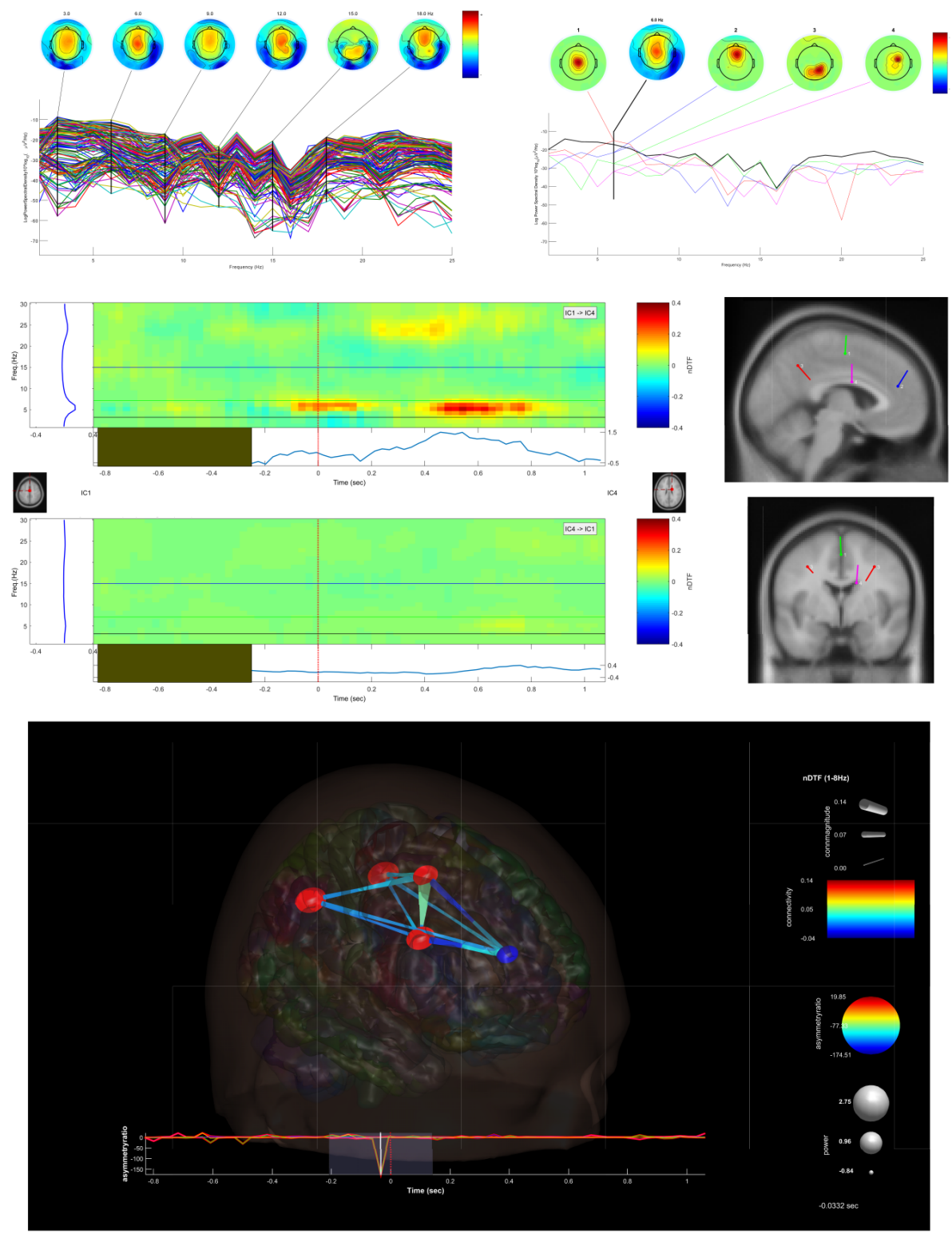

Figure 2 Functional connectivity analysis. Example of a preliminary test of connectivity analysis on a sample of 20 healthy individuals administered with the somatosensory repeated stimulation mentioned in the current project. The left plot in the upper panel shows the frequency spectrum of EEG power $(1-25 \mathrm{~Hz})$ across single trials in the sample. The plot on the right provides independent components (as specified by the independent component analysis) of the EEG power at $6 \mathrm{~Hz}$. This activity is well-known to represent the vertex EEG response commonly elicited by salient sensory stimuli. The left plot in the centre panel shows the main result of the functional connectivity analysis (as performed by EEGLAB): The normalized direct transfer function (nDTF) reveals an increased connectivity from the first component (IC1) to the fourth component (IC4) in the target frequency (peaking at $6 \mathrm{~Hz}$ and $\approx 500$ ms poststimulus relative to baseline). Dipole modelling of the ICs (centre right) localizes IC1 and IC4 at Brodmann areas 6 (premotor or supplementary motor area, green dipole) and 24 (medial portion of the anterior cingulate cortex). The bottom panel figure extends this analysis by indicating an inflow from IC4 to IC1 as well as reduced flow between these two ICS and the second IC located in the prefrontal region. This pattern takes place right before the arrival of the somatosensory stimulus. Altogether, these preliminary findings hint at a potential twofold pattern of pre-post stimulus connectivity between these regions that could be considered as a small world network for the identification of a purported neurological marker in fibromyalgia patients. 
Figure $\mathbf{2}$ (Cont.) The left plot in the centre panel shows the main result of the functional connectivity analysis (as performed by EEGLAB): The normalized direct transfer function (nDTF) reveals an increased connectivity from the first component (IC1) to the fourth component (IC4) in the target frequency (peaking at $6 \mathrm{~Hz}$ and $\approx 500$ ms poststimulus relative to baseline). Dipole modelling of the ICs (centre right) localizes IC1 and IC4 at Brodmann areas 6 (premotor or supplementary motor area, green dipole) and 24 (medial portion of the anterior cingulate cortex). The bottom panel figure extends this analysis by indicating an inflow from IC4 to IC1 as well as reduced flow between these two ICS and the second IC located in the prefrontal region. This pattern takes place right before the arrival of the somatosensory stimulus. Altogether, these preliminary findings hint at a potential twofold pattern of pre-post stimulus connectivity between these regions that could be considered as a small world network for the identification of a purported neurological marker in fibromyalgia patients.

\section{Cost estimates}

Total budget requested

$£ 99,555.00$

Intended starting date

29/5/2019

\section{Application for additional grants}

This application has been revised and resubmitted to the Wellcome Trust Seed Grant Round 2019 (but no feedback is provided in that scheme).

\section{Data management plan}

The project will generate different types of data that can be measured on ordinal and interval scales, and in different numerical formats. Self-report data will be electronically collected through specific software (e.g., E-prime, Qualtrics) and exported to statistics software format (.sav in SPSS or .cvs for more sophisticated analyses in R open source software). Other data formats include.cnt (Neuroscan EEG recording) and set (EEGlab software) for processing of EEG activity. Cytokine concentration will be recorded from readings of absorbance (in nanometers) and stored in digital format. They will be of value for other researchers in the field of pain, particularly those with a background in clinical neuroscience. Data quality will be checked to ensure that the scientific community can be warned about any missing, excluded, or compromised data. The post-doctoral researcher and all technical staff will receive detailed guidance in best practices of task administration, data collection, and storage to guarantee that data is of consistent high quality. Dr. Metodiev and I will ultimately be responsible for ensuring the proper management of the data; we will supervise all computations and verify statistics to ensure accuracy. Data will be backed-up on a cloud server (e.g., OneDrive) in compliance with the European Data Protection Act. The University of Essex's network is secure and protected from viruses and intrusions by a firewall and the Sophos anti-virus program. The consent forms will be stored and locked in our offices at the University. Data will then be shared upon completion of data collection and before data analysis. As I previously mentioned, data will be made available on the Open Science Framework (OSF) as well as on the University of Essex Research Reposi- tory (http://repository.essex.ac.uk/), so they will be openly accessible to other researchers. Individual data will be anonymized and stored on passwordprotected computers.

\section{Ethical aspects}

I have had 3 different ethics applications being approved by the University of Essex so far and all of them implied the use of nociceptive stimulation and induction of tolerable pain (EV1701, EV1501, EV1801). The ethics approval procedure entails the submission of different forms to the departmental Ethics Officer. Applications will first be assessed by the Ethics Officer before being passed on to the University's Ethics Committee. A copy of my research proposal and any necessary supporting documentation (e.g., consent form, recruiting materials, etc.) will be attached and will be assessed by the Research Governance and Planning Manager. Being familiar with this procedure, I expect to submit the ethics application for the present project at the end of the current year and for the approval process to take no longer than two months. Therefore, I foresee receiving full approval by the end of March 2019.

\section{Declarations}

Head of department's statement of support I fully support this application. Fibromyalgia is a very poorly understood, under-researched condition with a huge impact on quality of life. Understanding the organic underpinnings of this chronic pain condition is important for both establishing the nature of the conditions and developing interventions. Dr. Valentini's expertise in pain will provide vital insights into the biomarkers of this condition by combining EEG and cytokine analysis. His expertise, which he will continue to develop through this project, will position him excellently for this. He is unusual in combining a strong physiological background with a well-rounded understanding of the person as a whole, and the subjective experience in this condition of chronic pain. Elia has recently become a permanent member of the department, having established his research here very successfully, combined with being an excellent teacher and valuable colleague. The university has invested significantly in the infrastructure required for research in pain, 
and in the appropriate EEG techniques. Elia has matched this with his own investment in getting this facility up and running safely and successfully and securing funding for a PhD student. This continued investment will diversify his skills and represent long-term commitment to establishing his excellent research program and academic career.

Society

Public summary

Lay summary Fibromyalgia (FM) is one of the most disabling chronic pain conditions. Science is still struggling to understand how it develops and how to treat it. More research on the basic mechanisms of this disease is needed. We currently know that FM patients show imbalanced brain activity and produce a range of neural chemicals associated with response to stress and inflammation, but we do not know how to use this information to discriminate between patients who positively respond to treatment and those who do not. The lack of effective treatments has led clinical practitioners to call for more research addressing the development of the disease and the effectiveness of treatment.

The goal of this project is to identify robust neurological measures of FM (i.e., electrochemical brain activity) by applying a combination of advanced analyses of electrical brain activity and inflammatory brain responses in FM patients who experience successful vs. unsuccessful treatment (compared to healthy individuals). The University of Essex is perfectly suited in terms of expertise and facilities to identify the electrical brain responses during rest and sensory stimulation, as well as measuring chemical stress responses at the same time.

This project is important because the successful identification of specific cerebral measures of FM can improve the assessment of the development of this disease and pave the way to study its relationship with measures of treatment effectiveness. This research could significantly impact patients' prospects of better identification and treatment of this chronic pain condition.

\section{Reviews}

See supplemental file 1.

\section{Rebuttal}

Not available

\section{Decision}

See supplemental file 2 .

\section{References}

[1] Fayaz, A., Croft, P., Langford, R. M., Donaldson, L. J., \& Jones, G. T. (2016). Prevalence of chronic pain in the UK: A systematic review and meta-analysis of population studies. BMJ Open, 6(6), e010364. https: //doi.org/10.1136/bmjopen-2015-010364

[2] Breivik, H., Collett, B., Ventafridda, V., Cohen, R., \& Gallacher, D. (2006). Survey of chronic pain in Europe: Prevalence, impact on daily life, and treatment. European Journal of Pain, 10(4), 287-287. https://doi. org/10.1016/j.ejpain.2005.06.009

[3] Clauw, D. J. (2014). Fibromyalgia: A Clinical Review. JAMA, 311(15), 1547. https://doi.org/10.1001/jama. 2014.3266

[4] Wolfe, F., Clauw, D. J., Fitzcharles, M.-A., Goldenberg, D. L., Katz, R. S., Mease, P., Russell, A. S., Russell, I. J., Winfield, J. B., \& Yunus, M. B. (2010). The American College of Rheumatology Preliminary Diagnostic Criteria for Fibromyalgia and Measurement of Symptom Severity. Arthritis Care \& Research, 62(5), 600610. https://doi.org/10.1002/acr.20140

[5] Walitt, B., Katz, R. S., Bergman, M. J., \& Wolfe, F. (2016) Three-Quarters of Persons in the US Population Reporting a Clinical Diagnosis of Fibromyalgia Do Not Satisfy Fibromyalgia Criteria: The 2012 National Health Interview Survey (C. Sommer, Ed.). PLOS ONE, 11(6), e0157235. https://doi.org/10.1371/journal. pone.0157235

[6] Sluka, K. A., \& Clauw, D. J. (2016). Neurobiology of fibromyalgia and chronic widespread pain. Neuroscience, 338, 114-129. https://doi.org/10.1016/j. neuroscience.2016.06.006

[7] Napadow, V., LaCount, L., Park, K., As-Sanie, S., Clauw, D. J., \& Harris, R. E. (2010). Intrinsic brain connectivity in fibromyalgia is associated with chronic pain intensity. Arthritis \& Rheumatism, 62(8), 2545-2555. https://doi.org/10.1002/art.27497

[8] Napadow, V., Kim, J., Clauw, D. J., \& Harris, R. E. (2012). Brief Report: Decreased intrinsic brain connectivity is associated with reduced clinical pain in fibromyalgia. Arthritis \& Rheumatism, 64(7), 2398-2403. https: //doi.org/10.1002/art.34412

[9] Chatterjea, D., \& Martinov, T. (2015). Mast cells: Versatile gatekeepers of pain. Molecular Immunology, 63(1), 38-44. https://doi.org/10.1016/j.molimm. 2014.03.001

[10] Theoharides, T. C., Tsilioni, I., Arbetman, L., Panagiotidou, S., Stewart, J. M., Gleason, R. M., \& Russell, I. J. (2015). Fibromyalgia Syndrome in Need of Effective Treatments. Journal of Pharmacology and Experimental Therapeutics, 355(2), 255-263. https: //doi.org/10.1124/jpet.115.227298

[11] Galic, M. A., Riazi, K., \& Pittman, Q.J. (2012). Cytokines and brain excitability. Frontiers in Neuroendocrinology, 33(1), 116-125. https://doi.org/10.1016/j.yfrne. 2011.12.002

[12] Lauche, R., Hauser, W., \& Jung, E. (2013). Patientrelated predictors of treatment satisfaction of patients with fibromyalgia syndrome: Results of a cross-sectional survey. Clinical and experimental rheumatology, 31

[13] Macfarlane, G., Kronisch, C., \& Dean, L. (2016). EU $L A R$ revised recommendations for the management of fibromyalgia. BMJ (Clinical research ed.), 1-11. https://doi.org/10.1136/annrheumdis 
[14] Carville, S. F., \& Choy, E. H. (2008). Systematic Review of Discriminating Power of Outcome Measures Used in Clinical Trials of Fibromyalgia. The Journal of Rheumatology, 35(11), 2094-2105. https://doi.org/ 10.3899/jrheum.080077

[15] Kroenke, K., \& Spitzer, R. L. (2002). The PHQ-9: A New Depression Diagnostic and Severity Measure. Psychiatric Annals, 32(9), 509-515. https://doi.org/ 10.3928/0048-5713-20020901-06

[16] Spitzer, R. L., Kroenke, K., Williams, J. B. W., \& Löwe, B. (2006). A brief measure for assessing generalized anxiety disorder: The GAD-7. Archives of Internal Medicine, 166(10), 1092-1097. https://doi.org/10. 1001/archinte.166.10.1092

[17] Sullivan, M. J. L., Bishop, S. R., \& Pivik, J. (1995). The Pain Catastrophizing Scale: Development and validation. Psychological Assessment, 7(4), 524-532. https: //doi.org/10.1037/1040-3590.7.4.524
[18] Tennant, R., Hiller, L., Fishwick, R., Platt, S., Joseph, S Weich, S., Parkinson, J., Secker, J., \& Stewart-Brown, S. (2007). The Warwick-Edinburgh Mental Well-being Scale (WEMWBS): Development and UK validation. Health and Quality of Life Outcomes, 5(1), 63. https: //doi.org/10.1186/1477-7525-5-63

[19] Legrain, V., Mancini, F., Sambo, C., Torta, D., Ronga, I., \& Valentini, E. (2012). Cognitive aspects of nociception and pain. Bridging neurophysiology with cognitive psychology. Neurophysiologie Clinique/Clinical Neurophysiology, 42(5), 325-336. https://doi.org/ 10.1016/j.neucli.2012.06.003

[20] Torta, D., Legrain, V., Mouraux, A., \& Valentini, E. (2017). Attention to pain! A neurocognitive perspective on attentional modulation of pain in neuroimaging studies. Cortex, 89, 120-134. https://doi.org/10. 1016/j.cortex.2017.01.010 